\title{
A SURVEY ON BESSEL TYPE FUNCTIONS AS MULTI-INDEX MITTAG-LEFFLER FUNCTIONS: DIFFERENTIAL AND INTEGRAL RELATIONS
}

\author{
Jordanka Paneva-Konovska ${ }^{1,2}$ \\ ${ }^{1}$ Faculty of Applied Mathematics and Informatics \\ Technical University of Sofia \\ 8 Kliment Ohridski, Sofia - 1000, BULGARIA \\ ${ }^{2}$ Institute of Mathematics and Informatics \\ Bulgarian Academy of Sciences \\ 'Acad. G. Bontchev' Street, Block 8 \\ Sofia - 1113, BULGARIA
}

\begin{abstract}
As recently observed by Bazhlekova and Dimovski [1], the $n$ th derivative of the 2-parametric Mittag-Leffler function gives a 3-parametric Mittag-Leffler function, known as the Prabhakar function. Following the analogy, the $n$-th derivatives of the Bessel type functions are obtained, and it turns out that usually they are expressed in terms of the Bessel type functions with the same or more number of indices, up to a matching power function. Further, some special cases of the fractional order Riemann-Liouville derivatives and integrals of the Bessel type functions are calculated and interesting relations are proved.
\end{abstract}

AMS Subject Classification: 33E12, 33C10, 26A33

Key Words: Bessel functions and generalizations, Mittag-Leffler functions, multi-index Mittag-Leffler functions, fractional calculus, Riemann-Liouville fractional integral and derivative

Received: March 3, 2019

(C) 2019 Academic Publications 


\section{Introduction}

This is a survey paper on a part of author's publications, referring to the differentiation and integration of an arbitrary order of the Bessel type functions. In the beginning we consider both the classical Bessel functions of the first kind and the Bessel-Clifford functions, which are closely related. The first are defined by the series

$$
J_{\nu}(z)=\sum_{k=0}^{\infty} \frac{(-1)^{k}(z / 2)^{2 k+\nu}}{k ! \Gamma(k+\nu+1)}, \quad z \in \mathbb{C} \backslash(-\infty, 0] ; \nu \in \mathbb{C},
$$

and the second, which are entire functions, are defined by the power series

$$
C_{\nu}(z)=\sum_{k=0}^{\infty} \frac{(-1)^{k}(z)^{k}}{k ! \Gamma(k+\nu+1)}, \quad z \in \mathbb{C} ; \nu \in \mathbb{C} .
$$

Originating from concrete problems in mechanics and astronomy, these functions have plenty of applications. In that reason they both have various generalizations with more indices (parameters).

In contrast, the Mittag-Leffler functions, which are entire functions, defined by the power series

$$
E_{\alpha, \beta}(z)=\sum_{k=0}^{\infty} \frac{z^{k}}{\Gamma(\alpha k+\beta)} \quad(z \in \mathbb{C} ; \alpha, \beta \in \mathbb{C}, \operatorname{Re}(\alpha)>0),
$$

originated from a purely theoretical problem connected with an analytical continuation of a function. Arising in the beginning of 20th century (initially as $E_{\alpha}(z)$, for $\beta=1$ ) they remained unremarked and unused for a long time (almost for a half of century). Nevertheless, nowadays they also have numerous generalizations, related to applications in Fractional Calculus (FC) and fractional order differential equations and systems.

\section{Generalizations of the Bessel functions}

Here we consider the generalizations of the Bessel and Bessel-Clifford functions with two, three and four indices, or briefly said Bessel type functions. We begin with the entire functions

$$
J_{\nu}^{\mu}(z)=\sum_{k=0}^{\infty} \frac{(-z)^{k}}{k ! \Gamma(\mu k+\nu+1)}, \quad z \in \mathbb{C} ; \nu \in \mathbb{C} \text { and } \mu>-1,
$$

introduced by the British mathematician Sir Edward Maitland Wright [26] and known in the literature as Bessel-Maitland functions (named after his second name). Wright firstly introduced them for $\mu>0$ and on a later stage, studying the asymptotic behavior of the Bessel-Maitland functions 
for large values of $|z|$, he extended their definition for $\mu>-1$ ([27], see also [13] and [14]). Let us mention, that for $\mu=1$ the Bessel-Maitland function becomes Bessel-Clifford function, i.e.

$$
C_{\nu}(z)=J_{\nu}^{1}(z)
$$

We also consider the generalized Bessel-Maitland (or Bessel-Wright) functions with 3 parameters (introduced by R.S. Pathak [22]):

$$
\begin{array}{r}
J_{\nu, \sigma}^{\mu}(z)=(z / 2)^{\nu+2 \sigma} \sum_{k=0}^{\infty} \frac{(-1)^{k}(z / 2)^{2 k}}{\Gamma(k+\sigma+1) \Gamma(\mu k+\sigma+\nu+1)}, \\
z \in \mathbb{C} \backslash(-\infty, 0] ; \quad \nu, \sigma \in \mathbb{C}, \quad \mu>0,
\end{array}
$$

and along with it

$$
\begin{array}{r}
J_{\nu, \sigma}^{\mu}(2 \sqrt{z})=z^{\nu / 2+\sigma} \sum_{k=0}^{\infty} \frac{(-z)^{k}}{\Gamma(k+\sigma+1) \Gamma(\mu k+\sigma+\nu+1)}, \\
z \in \mathbb{C} \backslash(-\infty, 0] ; \quad \nu, \sigma \in \mathbb{C}, \quad \mu>0 .
\end{array}
$$

At last, the 4-index generalizations (Prieto and all [24]) are given by the formula

$$
\begin{array}{r}
J_{\nu, \sigma}^{\mu, m}(z)=(z / 2)^{\nu+2 \sigma} \sum_{k=0}^{\infty} \frac{(-1)^{k}(z / 2)^{2 k}}{(\Gamma(k+\sigma+1))^{m} \Gamma(\mu k+\sigma+\nu+1)}, \\
z \in \mathbb{C} \backslash(-\infty, 0] ; \quad \nu, \sigma \in \mathbb{C}, \quad \mu>0, \quad m \in \mathbb{N},
\end{array}
$$

and along with it

$$
\begin{array}{r}
J_{\nu, \sigma}^{\mu, m}(2 \sqrt{z})=z^{\nu / 2+\sigma} \sum_{k=0}^{\infty} \frac{(-z)^{k}}{(\Gamma(k+\sigma+1))^{m} \Gamma(\mu k+\sigma+\nu+1)}, \\
z \in \mathbb{C} \backslash(-\infty, 0] ; \quad \nu, \sigma \in \mathbb{C}, \quad \mu>0, \quad m \in \mathbb{N} .
\end{array}
$$

It is easily seen there is a simple relation between the two-index BesselMaitland functions $J_{\nu}^{\mu}(z)$ and the generalized Bessel-Maitland functions with 3 indices, namely

$$
J_{\nu}^{\mu}(z)=z^{-\nu / 2} J_{\nu, 0}^{\mu}(2 \sqrt{z}), \quad z \neq 0 ; \nu \in \mathbb{C}, \mu>0
$$

and in particular, for $\mu=1$ the relation is between $C_{\nu}$ and $J_{\nu, 0}^{1}=J_{\nu}$, i.e.

$$
C_{\nu}(z)=z^{-\nu / 2} J_{\nu}(2 \sqrt{z}), \quad z \neq 0, \nu \in \mathbb{C} .
$$

Studying the properties of the functions, mentioned above, integrals and derivatives of an arbitrary order of them are found. As a result, different interesting relations between these integrals and derivatives and functions of the considered kind are often obtained. The corresponding special cases for the Bessel functions of the first kind (1), Bessel-Clifford functions (2), and Mittag-Leffler functions (3), merely follow as corollaries. Such type of results are also obtained for other Special Functions of Fractional Calculus, for more 
general functions see e.g. [7]. Various useful results for the generalized fractional calculus operators of special functions are given in the interesting paper [11]. A part of the results connected with the 2-parametric Bessel-Maitland functions are directly obtained by the author in [21]. Other type of properties for the functions families of the kinds (3) and (11) can be seen in the survey papers [17] and [18] by the author.

\section{Generalizations of the Mittag-Leffler functions}

Regardless of their purely theoretical origin, nowadays the Mittag-Leffler functions enjoy many generalizations and wide applications. Among them is the 3-parametric Mittag-Leffler function

$$
E_{\alpha, \beta}^{\gamma}(z)=\sum_{k=0}^{\infty} \frac{(\gamma)_{k}}{\Gamma(\alpha k+\beta)} \frac{z^{k}}{k !} \quad(z \in \mathbb{C} ; \alpha, \beta, \gamma \in \mathbb{C}, \operatorname{Re}(\alpha)>0),
$$

introduced by Prabhakar [23] and known in the literature also as the Prabhakar function. It is clear that

$$
E_{\alpha, \beta}^{1}(z)=E_{\alpha, \beta}(z), \quad E_{\alpha, 1}^{1}(z)=E_{\alpha, 1}(z)=E_{\alpha}(z) .
$$

Other generalizations are the multi-index Mittag-Leffler functions. First of them is the multi-index Mittag-Leffler function with $2 m$ parameters ( $m=$ $1,2,3, \ldots)$ :

$$
E_{\left(\alpha_{i}\right),\left(\beta_{i}\right)}(z)=E_{\left(\alpha_{i}\right),\left(\beta_{i}\right)}^{m}(z)=\sum_{k=0}^{\infty} \frac{z^{k}}{\Gamma\left(\alpha_{1} k+\beta_{1}\right) \ldots \Gamma\left(\alpha_{m} k+\beta_{m}\right)} .
$$

It was introduced by Luchko and Yakubovich [28] and Kiryakova [8] and studied in details by Kiryakova [8, 10]. Originally defined for $\alpha_{i}>0$ and $\beta_{i}$ arbitrary real (complex) numbers, later its definition is extended for complex parameters with $\operatorname{Re}\left(\alpha_{i}\right)>0$ (see e.g. [8] for the first case and the works [3, 4] for the second one). For the applications of this class of special functions in the solutions of fractional order differential equations and models, see e.g. in Kiryakova and Luchko [12]. The survey by Kilbas et al. [5] describes the historical development of the theory of these multi-index (2m-parametric) Mittag-Leffler functions as a subclass of the Wright generalized hypergeometric functions ${ }_{p} \Psi_{q}(z)$. The method of Mellin-Barnes type integral representations allows these authors to extend the considered functions and to study them in the case of arbitrary values of parameters.

The next is the multi-index Mittag-Leffler function defined by means of $3 \mathrm{~m}$ parameters: 


$$
\begin{gathered}
E_{\left(\alpha_{i}\right),\left(\beta_{i}\right)}^{\left(\gamma_{i}\right)}(z)=E_{\left(\alpha_{i}\right),\left(\beta_{i}\right)}^{\left(\gamma_{i}\right), m}(z)=\sum_{k=0}^{\infty} \frac{\left(\gamma_{1}\right)_{k} \ldots\left(\gamma_{m}\right)_{k}}{\Gamma\left(\alpha_{1} k+\beta_{1}\right) \ldots \Gamma\left(\alpha_{m} k+\beta_{m}\right)} \frac{z^{k}}{(k !)^{m}} \\
\left(z \in \mathbb{C} ; \alpha_{i}, \beta_{i}, \gamma_{i} \in \mathbb{C}, \operatorname{Re}\left(\alpha_{i}\right)>0\right),
\end{gathered}
$$

and was introduced by the author in $[16,19]$. It generalizes both the 3 parametric Prabhakar function (11) and the $2 m$-parametric Mittag-Leffler function (12).

More precisely, taking $m=1$ in (13), the Prabhakar function (11) is produced. If $\gamma_{1}=\cdots=\gamma_{m}=1$, then (13) is reduced to the $2 m$-parametric function (12).

All of these listed functions can be expressed by the more general Wright generalized hypergeometric functions ${ }_{p} \Psi_{q}$ :

$$
\left.{ }_{p} \Psi_{q}\left[\begin{array}{c}
\left(a_{1}, A_{1}\right) \ldots\left(a_{p}, A_{p}\right) \\
\left(b_{1}, B_{1}\right) \ldots\left(b_{q}, B_{q}\right)
\end{array}\right) \sigma\right]=\sum_{k=0}^{\infty} \frac{\Gamma\left(a_{1}+k A_{1}\right) \ldots \Gamma\left(a_{p}+k A_{p}\right)}{\Gamma\left(b_{1}+k B_{1}\right) \ldots \Gamma\left(b_{q}+k B_{q}\right)} \frac{\sigma^{k}}{k !},
$$

with $A_{i}>0, B_{i}>0, a_{i}, b_{i} \in \mathbb{C}, i=1, \ldots, m$.

\section{The Bessel type functions as multi-index Mittag-Leffler functions}

The Bessel functions and their generalizations are also connected with the multiindex Mittag-Leffler functions. The relations between them are given in this section.

Again, let $\gamma_{1}=\cdots=\gamma_{m}=1$. A special case of (13) (for $m \geq 2$ ) is the generalized Lommel-Wright function with 4 indices $(\mu>0, q \in \mathbb{N}, \nu, \lambda \in \mathbb{C})$, introduced by de Oteiza, Kalla and Conde (for details see [9]):

This is an interesting example of a multi-index Mittag-Leffler function with arbitrary $m=q+1, q \in \mathbb{N}$. It is clear that for $\alpha_{1}=\mu, \alpha_{2}=1, \ldots, \alpha_{q+1}=1$, $\beta_{1}=\sigma+\nu+1, \beta_{2}=\sigma+1, \ldots, \beta_{q+1}=\sigma+1$, the generalized Lommel-Wright function can be expressed by the multi-index Mittag-Leffler functions (12) and (13) as follows:

$$
\begin{array}{r}
J_{\nu, \sigma}^{\mu, q}(z)=(z / 2)^{\nu+2 \sigma} E_{(\mu, 1, \ldots, 1),(\sigma+\nu+1, \sigma+1, \ldots, \sigma+1)}^{(1, \ldots, 1), q+1}\left(-\frac{z^{2}}{4}\right) \\
=(z / 2)^{\nu+2 \sigma} E_{(\mu, 1, \ldots, 1),(\sigma+\nu+1, \sigma+1, \ldots, \sigma+1)}^{q+1}\left(-\frac{z^{2}}{4}\right), \\
z \in \mathbb{C} \backslash(-\infty, 0] ; \quad \nu, \sigma \in \mathbb{C}, \quad \mu>0,
\end{array}
$$


respectively

$$
\begin{array}{r}
J_{\nu, \sigma}^{\mu, q}(2 \sqrt{z})=z^{\nu / 2+\sigma} E_{(\mu, 1, \ldots, 1),(\sigma+\nu+1, \sigma+1, \ldots, \sigma+1)}^{(1, \ldots, 1), q+1}(-z) \\
=z^{\nu / 2+\sigma} E_{(\mu, 1, \ldots, 1),(\sigma+\nu+1, \sigma+1, \ldots, \sigma+1)}^{q+1}(-z), \\
z \in \mathbb{C} \backslash(-\infty, 0] ; \quad \nu, \sigma \in \mathbb{C}, \quad \mu>0 .
\end{array}
$$

Further, for $q=1, \alpha_{1}=\mu, \alpha_{2}=1, \beta_{1}=\sigma+\nu+1, \beta_{2}=\sigma+1$, the equalities (15) and (16) give:

$$
\begin{aligned}
J_{\nu, \sigma}^{\mu}(z) & =(z / 2)^{\nu+2 \sigma} E_{(\mu, 1),(\sigma+\nu+1, \sigma+1)}^{(1,1)}\left(-\frac{z^{2}}{4}\right) \\
& =(z / 2)^{\nu+2 \sigma} E_{(\mu, 1),(\sigma+\nu+1, \sigma+1)}\left(-\frac{z^{2}}{4}\right), \\
z & \in \mathbb{C} \backslash(-\infty, 0] ; \quad \nu, \sigma \in \mathbb{C}, \quad \mu>0,
\end{aligned}
$$

respectively

$$
\begin{aligned}
& J_{\nu, \sigma}^{\mu}(2 \sqrt{z})=z^{\nu / 2+\sigma} E_{(\mu, 1),(\sigma+\nu+1, \sigma+1)}^{(1,1)}(-z) \\
&=z^{\nu / 2+\sigma} E_{(\mu, 1),(\sigma+\nu+1, \sigma+1)}(-z), \\
& z \in \mathbb{C} \backslash(-\infty, 0] ; \quad \nu, \sigma \in \mathbb{C}, \quad \mu>0 .
\end{aligned}
$$

Additionally, if $\sigma=0$, i. e. for $q=1, \alpha_{1}=\mu, \alpha_{2}=1, \beta_{1}=\nu+1, \beta_{2}=1$, the relations (15) and (17) produce:

$$
\begin{array}{r}
J_{\nu}^{\mu}(z)=E_{(\mu, 1),(\nu+1,1)}^{(1,1)}(-z)=E_{(\mu, 1),(\nu+1,1)}(-z), \\
z \in \mathbb{C} ; \nu \in \mathbb{C} \text { and } \mu>0,
\end{array}
$$

and finally, if $\alpha_{1}=\mu=1$, the formula (19) is reduced to

$$
C_{\nu}(z)=E_{(1,1),(\nu+1,1)}^{(1,1)}(-z)=E_{(1,1),(\nu+1,1)}(-z), \quad z \in \mathbb{C} ; \nu \in \mathbb{C} .
$$

Thus, all the considered functions in the preceding sections, both the generalizations of the Bessel and Mittag-Leffler functions, are expressed by the multi-index Mittag-Leffler functions (13) (and the most of them by (12)), not only by the Wright generalized hypergeometric functions (14). In what follows we essentially use these relations. 


\section{Integer order derivatives}

In order to continue our study, we firstly need some results for the integer order derivatives.

Recently, it has been obtained by Bazhlekova and Dimovski [1] that the $n$-th derivative of the 2-parametric Mittag-Leffler function gives a 3-parametric Mittag-Leffler function, introduced by Prabhakar (up to a constant), namely

$$
E_{\alpha, \beta}^{(n)}(z)=n ! E_{\alpha, \beta+n \alpha}^{n+1}(z) .
$$

Following the analogy, the $n$-th derivative of the generalized Bessel functions in Section 2 are calculated. For this purpose we are based on the following result giving the $n$-th derivative of the multi-index Mittag-Leffler function (13), multiplied by a suitable power function (for details and proofs see [16] and [19]).

Theorem 1. Let $z \in \mathbb{C} \backslash(-\infty, 0]$ be a complex variable and let $\alpha_{i}, \beta_{i}, \gamma_{i}, \omega \in$ $\mathbb{C}, \operatorname{Re}\left(\alpha_{i}\right)>0, \operatorname{Re}\left(\beta_{i_{0}}\right)>0, i=1, \ldots, m, 1 \leq i_{0} \leq m, i_{0} \in \mathbb{N}$, then for any $n \in \mathbb{N}$ the following identity holds:

$$
\begin{gathered}
D^{n}\left[z^{\beta_{i_{0}}-1} E_{\left(\alpha_{i}\right),\left(\beta_{i}\right)}^{\left(\gamma_{i}\right), m}\left(\omega z^{\alpha_{i_{0}}}\right)\right]=\left(\frac{d}{d z}\right)^{n}\left[z^{\beta_{i_{0}}-1} E_{\left(\alpha_{i}\right),\left(\beta_{i}\right)}^{\left(\gamma_{i}\right), m}\left(\omega z^{\alpha_{i_{0}}}\right)\right] \\
=z^{\beta_{i_{0}}-n-1} E_{\left(\alpha_{i}\right),\left(\widetilde{\beta}_{i}\right)}^{\left(\gamma_{i}\right), m}\left(\omega z^{\alpha_{i_{0}}}\right), \quad|\arg z|<\pi,
\end{gathered}
$$

with $\widetilde{\beta}_{i_{0}}=\beta_{i_{0}}-n$ and $\widetilde{\beta}_{i}=\beta_{i}$, if $i \neq i_{0}$.

Remark 2. In particular, as a result of (22), we obtain the following identities:

$$
\begin{gathered}
D^{n}\left[z^{\beta-1} E_{\alpha, \beta}^{\gamma}\left(\omega z^{\alpha}\right)\right]=z^{\beta-n-1} E_{\alpha, \beta-n}^{\gamma}\left(\omega z^{\alpha}\right), \\
D^{n}\left[z^{\beta_{i_{0}}-1} E_{\left(\alpha_{i}\right),\left(\beta_{i}\right)}^{m}\left(\omega z^{\alpha_{i}}\right)\right]=z^{\beta_{i_{0}}-n-1} E_{\left(\alpha_{i}\right),\left(\widetilde{\beta}_{i}\right)}^{m}\left(\omega z^{\alpha_{i_{0}}}\right),
\end{gathered}
$$

with $\widetilde{\beta}_{i_{0}}=\beta_{i_{0}}-n$ and $\widetilde{\beta}_{i}=\beta_{i}$, if $i \neq i_{0}$. These results follow, taking into account (11) and (12), if $m=1$, respectively, $\gamma_{1}=\cdots=\gamma_{m}=1$. The mentioned relations are well known, for which see Refs. [6] and [8].

The results below refer to the $n$-th derivatives of the generalized BesselLomel functions (7) and (8). It turns out that, in the most cases, these integer order derivatives are expressed by functions of the same kind, up to a constant. Further, due to the relation $J_{\nu, \sigma}^{\mu, 1}=J_{\nu, \sigma}^{\mu}$, the results obtained can be reduced 
to the 3-parametric generalized Bessel-Maitland functions (5) and (6), up to a power function.

Theorem 3. Let $z \in \mathbb{C} \backslash(-\infty, 0]$ be a complex variable, and let $\nu, \sigma \in \mathbb{C}$, $q \in \mathbb{N}$, be parameters. Then the following equalities hold true for all the values of $n \in \mathbb{N}_{0}$ :

$$
D^{n}\left[z^{-\sigma} J_{\nu, \sigma}^{2, q}(z)\right]=\frac{d^{n}}{d z^{n}}\left[z^{-\sigma} J_{\nu, \sigma}^{2, q}(z)\right]=2^{-n} z^{-\sigma} J_{\nu-n, \sigma}^{2, q}(z),
$$

for $\operatorname{Re}(\nu+\sigma+1)>0$,

$$
D^{n}\left[z^{\nu / 2} J_{\nu, \sigma}^{1, q}(2 \sqrt{z})\right]=z^{\nu / 2-n / 2} J_{\nu-n, \sigma}^{1, q}(2 \sqrt{z}),
$$

for $\operatorname{Re}(\nu+\sigma+1)>0$, and

$$
D^{n}\left[z^{-\nu / 2} J_{\nu, \sigma}^{\mu, q}(2 \sqrt{z})\right]=z^{\sigma-n} E_{(\mu, 1, \ldots, 1),(\sigma+\nu+1, \sigma-n+1, \sigma+1, \ldots, \sigma+1)}^{q+1}(-z),
$$

with $\operatorname{Re}(\sigma+1)>0$.

Proof. In what follows we denote for convenience

$$
\begin{aligned}
& \widetilde{J}_{\nu, \sigma}^{\mu, q}(z)=(z / 2)^{-\nu-2 \sigma} J_{\nu, \sigma}^{\mu, q}(z), \quad z \neq 0, \\
& \widetilde{J}_{\nu, \sigma}^{\mu, q}(2 \sqrt{z})=z^{-\nu / 2-\sigma} J_{\nu, \sigma}^{\mu, q}(2 \sqrt{z}), \quad z \neq 0 .
\end{aligned}
$$

Let us start with proving (24). Using the first relation (27), the function $z^{-\sigma} J_{\nu, \sigma}^{2, q}(z)$ can be represented as follows

$$
z^{-\sigma} J_{\nu, \sigma}^{2, q}(z)=z^{-\sigma}(z / 2)^{\nu+2 \sigma} \widetilde{J}_{\nu, \sigma}^{2, q}(z)=2^{-\nu-2 \sigma} z^{\nu+\sigma} \widetilde{J}_{\nu, \sigma}^{2, q}(z) .
$$

Then, in view of (15), applying the formula (22) with $\omega=-1 / 4, \alpha_{i_{0}}=2$, and the corresponding $\beta_{i_{0}}=\sigma+\nu+1$, we obtain

$$
D^{n}\left[z^{\sigma+\nu} \widetilde{J}_{\nu, \sigma}^{2, q}(z)\right]=z^{\sigma+\nu-n} \widetilde{J}_{\nu-n, \sigma}^{2, q}(z)=\frac{z^{-\sigma}}{2^{-(\nu+2 \sigma)} 2^{n}} J_{\nu-n, \sigma}^{2, q}(z) .
$$

Now, using (28), the equality (24) immediately follows.

Further, for proving (25), we express $z^{\nu / 2} J_{\nu, \sigma}^{1, q}(2 \sqrt{z})$ in an analogical way, and we obtain:

$$
z^{\nu / 2} J_{\nu, \sigma}^{1, q}(2 \sqrt{z})=z^{\nu+\sigma} \widetilde{J}_{\nu, \sigma}^{1, q}(2 \sqrt{z}) .
$$

Then, in view of (16) with $\omega=-1, \alpha_{i_{0}}=1$, and $\beta_{i_{0}}=\sigma+\nu+1$, we can write down that

$$
D^{n}\left[z^{\sigma+\nu} \widetilde{J}_{\nu, \sigma}^{1, q}(2 \sqrt{z})\right]=z^{\sigma+\nu-n} \widetilde{J}_{\nu-n, \sigma}^{1, q}(2 \sqrt{z})=z^{\nu / 2-n / 2} J_{\nu-n, \sigma}^{1, q}(2 \sqrt{z}),
$$


from where

$$
D^{n}\left[z^{\nu / 2} J_{\nu, \sigma}^{1, q}(2 \sqrt{z})\right]=z^{\sigma+\nu-n} \widetilde{J}_{\nu-n, \sigma}^{1, q}(2 \sqrt{z})=z^{\nu / 2-n / 2} J_{\nu-n, \sigma}^{1, q}(2 \sqrt{z}) .
$$

In order to prove (26), we apply the formula (23) for $\omega=-1, \alpha_{i_{0}}=1$, and the corresponding $\beta_{i_{0}}=\sigma+1$. Then, following the same idea as above, we obtain consequently

$$
\begin{gathered}
z^{-\nu / 2} J_{\nu, \sigma}^{\mu, q}(2 \sqrt{z})=z^{-\nu / 2} z^{\nu / 2+\sigma} \widetilde{J}_{\nu, \sigma}^{\mu, q}(2 \sqrt{z})=z^{\sigma} \widetilde{J}_{\nu, \sigma}^{\mu, q}(2 \sqrt{z}), \\
=z^{\sigma} \sum_{k=0}^{\infty} \frac{(-z)^{k}}{\Gamma(\mu k+\sigma+\nu+1)(\Gamma(k+\sigma+1))^{q}} .
\end{gathered}
$$

Therefore

$$
\begin{gathered}
D^{n}\left[z^{-\nu / 2} J_{\nu, \sigma}^{\mu, q}(2 \sqrt{z})\right]=D^{n}\left[z^{\sigma} \widetilde{J}_{\nu, \sigma}^{\mu, q}(2 \sqrt{z})\right] \\
=z^{\sigma-n} \sum_{k=0}^{\infty} \frac{(-z)^{k}}{\Gamma(\mu k+\sigma+\nu+1) \Gamma(k+\sigma-n+1)(\Gamma(k+\sigma+1))^{q-1}} \\
=z^{\sigma-n} E_{(\mu, 1, \ldots, 1),(\sigma+\nu+1, \sigma-n+1, \sigma+1, \ldots, \sigma+1)}^{q+1}(-z),
\end{gathered}
$$

which proves (26).

Remark 4. Putting $q=1$ in the equalities (24), (25) and (26), they give the corresponding results for the functions (5) and (6).

Corollary 5. Let $z \in \mathbb{C} \backslash(-\infty, 0]$ be a complex variable and let $\nu \in \mathbb{C}$ be a parameter with $\operatorname{Re}(\nu+1)>0$. Then the following equalities hold true for all the values of $n \in \mathbb{N}_{0}$ :

$$
D^{n}\left[z^{\nu / 2} J_{\nu}(2 \sqrt{z})\right]=z^{\nu / 2-n / 2} J_{\nu-n, 0}^{1,1}(2 \sqrt{z})=z^{\nu / 2-n / 2} J_{\nu-n}(2 \sqrt{z}) .
$$

Proof. The truthfulness of (33) immediately follows from (25), taking $q=1$ and $\sigma=0$.

Theorem 6. Let $z \in \mathbb{C} \backslash(-\infty, 0]$ be a complex variable and let $\mu, \nu$, be the parameters, satisfying the conditions $\mu>0, \nu \in \mathbb{C}$. Then the following equalities hold true for all the values of $n \in \mathbb{N}_{0}$ : 


$$
D^{n}\left[J_{\nu}^{\mu}(z)\right]=z^{-(\nu+n) / 2} J_{\nu+n,-n}^{\mu}(2 \sqrt{z}),
$$

and

$$
D^{n}\left[z^{\nu} C_{\nu}(z)\right]=z^{\nu-n} C_{\nu-n}(z) \quad \text { with } \quad \operatorname{Re}(\nu+1)>0 .
$$

Proof. Bearing in mind that $J_{\nu}^{\mu}(z)$ can be obtained from (12) taking $\alpha_{1}=1$, $\beta_{1}=1, \alpha_{2}=\mu$ and $\beta_{2}=\nu+1$, we apply (23) with $\alpha_{i_{0}}=1, \beta_{i_{0}}=1$ and $\omega=-1$. Before proving (34), we firstly note that its left-hand-side can also be written in the next form:

$$
\begin{aligned}
D^{n}\left[J_{\nu}^{\mu}(z)\right] & =z^{-n} \sum_{k=0}^{\infty} \frac{(-z)^{k}}{\Gamma(k-n+1) \Gamma(\mu k+\nu+1)} \\
& =z^{-n} \widetilde{J}_{\nu+n,-n}^{\mu}(2 \sqrt{z}) .
\end{aligned}
$$

Then, in view of (27), it follows

$$
D^{n}\left[J_{\nu}^{\mu}(z)\right]=z^{-(\nu+n) / 2} J_{\nu+n,-n}^{\mu}(2 \sqrt{z}),
$$

which proves (34).

Further putting $\mu=1$ in $J_{\nu}^{\mu}$ leads to $C_{\nu}$. Applying after that the second formula (23) with $\alpha_{i_{0}}=1$ and $\beta_{i_{0}}=\nu+1$ leads to the following result

$$
\begin{aligned}
& D^{n}\left[z^{\nu} C_{\nu}(z)\right]= z^{\nu-n} \sum_{\substack{k=0\\
}}^{\infty} \frac{(-z)^{k}}{\Gamma(k+1) \Gamma(k+\nu-n+1)} \\
&=z^{\nu-n} C_{\nu-n}(z) .
\end{aligned}
$$

Thus the correctness of (35) follows.

In particular, taking $\mu=1$ in (34), Theorem 6 gives result referring to the classical Bessel-Clifford functions, i.e. we produce the following corollary.

Corollary 7. Let the variable $z \in \mathbb{C} \backslash(-\infty, 0]$ and the parameter $\nu \in \mathbb{C}$. Then the following equalities hold true for all the values of $n \in \mathbb{N}_{0}$ and $z \neq 0$ :

$$
D^{n} C_{\nu}(z)=\left[C_{\nu}(z)\right]^{(n)}=z^{-(\nu+n) / 2} J_{\nu+n,-n}^{1}(2 \sqrt{z}) .
$$


Proof. Putting $\mu=1$, the function $J_{\nu}^{\mu}(z)$ becomes $C_{\nu}(z)$. Therefore the following logical conclusions can be deduced from (4) and (34):

$$
D^{n} C_{\nu}(z)=\left[C_{\nu}(z)\right]^{(n)}=\left[J_{\nu}^{1}(z)\right]^{(n)}=z^{-(\nu+n) / 2} J_{\nu+n,-n}^{1}(2 \sqrt{z}),
$$

which complete the proof.

Another interesting result, connecting the multi-index function (13) and the $n$-th derivative of (12) is formulated below.

Theorem 8. Let $\alpha_{i}, \beta_{i}, z \in \mathbb{C}$ and let $\operatorname{Re}\left(\alpha_{i}\right)>0$ for $i=1, \ldots, m$. Then the following equality holds true for all the values of $n \in \mathbb{N}_{0}$ :

with $\gamma_{1}=n+1, \gamma_{2}=\cdots=\gamma_{m}=1$.

$$
D^{n}\left[E_{\left(\alpha_{i}\right),\left(\beta_{i}\right)}(w z)\right]=w^{n} \Gamma(n+1) E_{\left(\alpha_{i}\right),\left(\beta_{i}+n \alpha_{i}\right)}^{\left(\gamma_{i}\right), m}(w z),
$$

Proof. The result has been proved for $w=1$ in [20]. The proof for (39) is completely similar (it can be also made as the $n$-th derivative of a composite function) and because of that details are omitted.

Remark 9. Taking $m=1$ and $w=1$, the equality (39) gives the relation (21), obtained by Bazhlekova and Dimovski in [1].

Further, the corresponding results for the 2-, 3-, and 4-index generalizations of the Bessel functions are given.

Theorem 10. Let $z$ be a complex variable and let $\mu, \nu, \sigma$ be the parameters, satisfying the conditions $\nu, \sigma \in \mathbb{C}, \mu>0$. Then the following equalities hold true for all the values of $n \in \mathbb{N}_{0}$ :

$$
D^{n}\left[z^{-\nu / 2-\sigma} J_{\nu, \sigma}^{\mu, q}(2 \sqrt{z})\right]=(-1)^{n} \Gamma(n+1) E_{\left(\alpha_{i}\right),\left(\widetilde{\beta}_{i}\right)}^{\left(\gamma_{i}\right), q+1}(-z),
$$

for $z \neq 0$ and with

$$
\begin{gathered}
\alpha_{1}=\mu, \alpha_{2}=\cdots=\alpha_{q+1}=1, \gamma_{1}=n+1, \gamma_{2}=\cdots=\gamma_{q+1}=1, \\
\widetilde{\beta}_{1}=\sigma+\nu+1+n \mu, \widetilde{\beta}_{2}=\cdots=\widetilde{\beta}_{q+1}=\sigma+n+1, \\
D^{n}\left[J_{\nu}^{\mu}(z)\right]=\frac{d^{n}}{d z^{n}}\left[J_{\nu}^{\mu}(z)\right]=(-1)^{n} J_{\nu+n \mu}^{\mu}(z), \quad z \in \mathbb{C},
\end{gathered}
$$

and

$$
D^{n}\left[C_{\nu}(z)\right]=\frac{d^{n}}{d z^{n}}\left[C_{\nu}(z)\right]=(-1)^{n} C_{\nu+n \mu}(z), \quad z \in \mathbb{C}
$$


Proof. Let us begin with the proof of (40). Using (16) and (39) with $w=$ -1 , along with it, we have

$$
\begin{gathered}
D^{n}\left[z^{-\nu / 2-\sigma} J_{\nu, \sigma}^{\mu, q}(2 \sqrt{z})\right]=D^{n}\left[E_{(\mu, 1, \ldots, 1),(\sigma+\nu+1, \sigma+1, \ldots, \sigma+1)}^{q+1}(-z)\right] \\
=(-1)^{n} \Gamma(n+1) E_{\left(\alpha_{i}\right),\left(\widetilde{\beta}_{i}\right)}^{\left(\gamma_{i}\right), q+1}(-z),
\end{gathered}
$$

with the same $\alpha_{i}, \widetilde{\beta}_{i}, \gamma_{i}$ as in $(41)(i=1, \ldots, q+1)$.

In order to prove (42), we use (19) and (39) with $w=-1$, and we obtain

$$
\begin{gathered}
D^{n} J_{\nu}^{\mu}(z)=D^{n} E_{(\mu, 1),(\nu+1,1)}(-z) \\
=(-1)^{n} \Gamma(n+1) E_{(\mu, 1),(n \mu+\nu+1, n+1)}^{(n+1,1), 2}(-z)=(-1)^{n} J_{\nu+n \mu}^{\mu}(z),
\end{gathered}
$$

which is exactly (42).

Now, if we put $\mu=1$ in the formula (42), it is reduced to (43).

Corollary 11. Let $z$ be a complex variable and let $\mu, \nu, q$ be the parameters, satisfying the conditions $\nu \in \mathbb{C}, \mu>0, q \in \mathbb{N}$. Then the following equalities hold true for all the values of $n \in \mathbb{N}_{0}$ :

$$
D^{n}\left[z^{-\nu / 2} J_{\nu, 0}^{\mu, q}(2 \sqrt{z})\right]=(-1)^{n} E_{\left(\alpha_{i}\right),\left(\widetilde{\beta}_{i}\right)}^{q+z)}(-z \neq 0,
$$

with

$$
\begin{gathered}
\alpha_{1}=\mu, \alpha_{2}=\cdots=\alpha_{q+1}=1 \\
\widetilde{\beta}_{1}=\nu+1+n \mu, \widetilde{\beta}_{2}=1+n, \cdots=\widetilde{\beta}_{q+1}=1+n .
\end{gathered}
$$

Proof. It immediately follows taking $\sigma=0$ in (40) and (41).

Remark 12. Putting $q=1$ in the equalities (40), and (46), they give the corresponding results for the three-parametric generalized Bessel-Maitland functions (6).

\section{Fractional Riemann-Liouville integral and derivative}

The most popular definition for integration of arbitrary (i.e. not obligatorily integer) order $\lambda \in \mathbb{C}(\operatorname{Re}(\lambda)>0)$ is the Riemann-Liouville $(R-L)$ fractional integral [25] 


$$
R^{\lambda} f(z)=\frac{1}{\Gamma(\lambda)} \int_{0}^{z}(z-t)^{\lambda-1} f(t) d t=\frac{z^{\lambda}}{\Gamma(\lambda)} \int_{0}^{1}(1-\tau)^{\lambda-1} f(z \tau) d \tau .
$$

The corresponding Riemann-Liouville fractional derivative of order $\lambda$ is defined as a composition of a derivative of integer order and an integral of fractional order of the form (48), namely:

$$
D^{\lambda} f(z):=D^{n} R^{n-\lambda} f(z)
$$

where $n:=[\operatorname{Re}(\lambda)]+1>\operatorname{Re}(\lambda),[\operatorname{Re}(\lambda)]=$ integer part of $\operatorname{Re}(\lambda)$.

For the theory and applications of the fractional Riemann-Liouville integral and derivative, as well as of other integral and differential operators of fractional calculus (FC), see the FC encyclopedia [25] and monograph [7], for the evolution and development of FC, see [15]. For miscellaneous useful applications of a number operators of FC, see also the recent survey paper [2].

Theorem 13. Let the variable $z \in \mathbb{C} \backslash(-\infty, 0]$ be a complex variable and let $\alpha_{i}, \beta_{i}, \gamma_{i}, \omega, \lambda \in \mathbb{C}, \operatorname{Re}\left(\alpha_{i}\right)>0, \operatorname{Re}\left(\beta_{i_{0}}\right)>0, \operatorname{Re}(\lambda)>0, i=1, \ldots, m$, $1 \leq i_{0} \leq m, i_{0} \in \mathbb{N}$. Then the following identity holds:

$$
R^{\lambda}\left[z^{\beta_{i_{0}}-1} E_{\left(\alpha_{i}\right),\left(\beta_{i}\right)}^{\left(\gamma_{i}\right), m}\left(\omega z^{\alpha_{i_{0}}}\right)\right]=z^{\beta_{i_{0}}+\lambda-1} E_{\left(\alpha_{i}\right),\left(\widetilde{\beta}_{i}\right)}^{\left(\gamma_{i}\right), m}\left(\omega z^{\alpha_{i}}\right),
$$

with $\widetilde{\beta}_{i_{0}}=\beta_{i_{0}}+\lambda$ and $\widetilde{\beta}_{i}=\beta_{i}$, if $i \neq i_{0}$.

Remark 14. In particular, as a result of (50), we obtain the following identities:

$$
\begin{gathered}
R^{\lambda}\left[z^{\beta-1} E_{\alpha, \beta}^{\gamma}\left(\omega z^{\alpha}\right)\right]=z^{\beta+\lambda-1} E_{\alpha, \beta+\lambda}^{\gamma}\left(\omega z^{\alpha}\right), \\
R^{\lambda}\left[z^{\beta_{i_{0}}-1} E_{\left(\alpha_{i}\right),\left(\beta_{i}\right)}^{m}\left(\omega z^{\alpha_{i_{0}}}\right)\right]=z^{\beta_{i_{0}}+\lambda-1} E_{\left(\alpha_{i}\right),\left(\widetilde{\beta}_{i}\right)}^{m}\left(\omega z^{\alpha_{i}}\right),
\end{gathered}
$$

with $\widetilde{\beta}_{i_{0}}=\beta_{i_{0}}+\lambda$ and $\widetilde{\beta}_{i}=\beta_{i}$, if $i \neq i_{0}$. These results follow, taking into account (11) and (12), if $m=1$ respectively $\gamma_{1}=\cdots=\gamma_{m}=1$. The mentioned relations are well known, for which see Refs. [6] and [8].

Using the relations in Section 4 along with Theorem 13, allows us to obtain the corresponding results connected with the functions of Bessel type. 
Theorem 15. Let $z \in \mathbb{C} \backslash(-\infty, 0]$ be a complex variable and let $\mu, \nu$ be the parameters, satisfying the conditions $\mu>0, \nu, \lambda \in \mathbb{C}$ and $\operatorname{Re}(\lambda)>0$. Then the following equalities hold true:

$$
\begin{gathered}
R^{\lambda}\left[z^{-\sigma} J_{\nu, \sigma}^{2, q}(z)\right]=2^{\lambda} z^{-\sigma} J_{\nu+\lambda, \sigma}^{2, q}(z), \\
R^{\lambda}\left[z^{\nu / 2} J_{\nu, \sigma}^{1, q}(2 \sqrt{z})\right]=z^{\nu / 2+\lambda / 2} J_{\nu+\lambda, \sigma}^{1, q}(2 \sqrt{z}),
\end{gathered}
$$

if additionally $\operatorname{Re}(\sigma+\nu+1)>0$. If $\operatorname{Re}(\sigma+1)>0$, then

$$
R^{\lambda}\left[z^{-\nu / 2} J_{\nu, \sigma}^{\mu, q}(2 \sqrt{z})\right]=z^{\sigma+\lambda} E_{(\mu, 1, \ldots, 1),(\sigma+\nu+1, \sigma+\lambda+1, \sigma+1, \ldots, \sigma+1)}^{q+1}(-z) .
$$

Proof. In view of (28) we can write

$$
z^{-\sigma} J_{\nu, \sigma}^{2, q}(z)=2^{-\nu-2 \sigma} z^{\nu+\sigma} \widetilde{J}_{\nu, \sigma}^{2, q}(z) .
$$

Applying after that the formula (50) for $\omega=-1 / 4, \alpha_{i_{0}}=2$, and the corresponding $\beta_{i_{0}}=\sigma+\nu+1$, we have

$$
R^{\lambda}\left[z^{\sigma+\nu} \widetilde{J}_{\nu, \sigma}^{2, q}(z)\right]=z^{\sigma+\nu+\lambda} \widetilde{J}_{\nu+\lambda, \sigma}^{2, q}(z)=\frac{2^{\lambda} z^{-\sigma}}{2^{-(\nu+2 \sigma)}} J_{\nu+\lambda, \sigma}^{2, q}(z) .
$$

Now, using (55), the equality (52) immediately follows. The proofs of (53) and (54) follow the same idea and they are omitted.

Remark 16. Putting $q=1$ in the equalities (52), (53) and (54), they give the corresponding results for the 3-index functions (5) and (6).

Corollary 17. Let $z \in \mathbb{C} \backslash(-\infty, 0]$ be a complex variable and let $\lambda, \nu \in \mathbb{C}$ be parameters, with $\operatorname{Re}(\lambda)>0$ and $\operatorname{Re}(\nu+1)>0$. Then the following equalities hold true

$$
R^{\lambda}\left[z^{\nu / 2} J_{\nu}(2 \sqrt{z})\right]=z^{\nu / 2+\lambda / 2} J_{\nu+\lambda, 0}^{1,1}(2 \sqrt{z})=z^{\nu / 2+\lambda / 2} J_{\nu+\lambda}(2 \sqrt{z}) .
$$

Proof. It follows by (53) taking $q=1$ and $\sigma=0$. 
Theorem 18. Let $z \in \mathbb{C} \backslash(-\infty, 0]$ be a complex variable and let $\lambda, \mu, \nu$ be the parameters, satisfying the conditions $\mu>0, \lambda, \nu \in \mathbb{C}, \operatorname{Re}(\lambda)>0$. Then the following equalities hold true

$$
R^{\lambda}\left[J_{\nu}^{\mu}(z)\right]=z^{-(\nu-\lambda) / 2} J_{\nu-\lambda, \lambda}^{\mu}(2 \sqrt{z})
$$

and

$$
R^{\lambda}\left[z^{\nu} C_{\nu}(z)\right]=z^{\nu+\lambda} C_{\nu+\lambda}(z) \quad \text { for } \quad \operatorname{Re}(\nu+1)>0
$$

Proof. Since $J_{\nu}^{\mu}(z)$ can be obtained from (12) taking $\alpha_{1}=1, \beta_{1}=1, \alpha_{2}=\mu$ and $\beta_{2}=\nu+1$, we apply the second formula (51) with $\alpha_{i_{0}}=1, \beta_{i_{0}}=1$ and $\omega=-1$. Before proving (57), we firstly note that its left-hand-side can also be presented as follows

$$
R^{\lambda}\left[J_{\nu}^{\mu}(z)\right]=z^{\lambda} \sum_{k=0}^{\infty} \frac{(-z)^{k}}{\Gamma(k+\lambda+1) \Gamma(\mu k+\nu+1)}=z^{\lambda} \widetilde{J}_{\nu-\lambda, \lambda}^{\mu}(2 \sqrt{z})
$$

Then, in view of (27), it follows

$$
R^{\lambda}\left[J_{\nu}^{\mu}(z)\right]=z^{-(\nu-\lambda) / 2} J_{\nu-\lambda, \lambda}^{\mu}(2 \sqrt{z})
$$

which proves (57).

Further putting $\mu=1$ in $J_{\nu}^{\mu}$ leads to $C_{\nu}$. Applying again the second formula (51) with $\alpha_{i_{0}}=1$ and $\beta_{i_{0}}=\nu+1$ leads to the following result

$$
R^{\lambda}\left[z^{\nu} C_{\nu}(z)\right]=z^{\nu+\lambda} \sum_{k=0}^{\infty} \frac{(-z)^{k}}{\Gamma(k+1) \Gamma(k+\nu+\lambda+1)}=z^{\nu+\lambda} C_{\nu+\lambda}(z) .
$$

Thus the correctness of (58) follows.

In particular, taking $\mu=1$, Theorem 18 gives a result referring to the classical Bessel-Clifford functions, i.e. we produce the following corollary.

Corollary 19. Let the variable $z \in \mathbb{C} \backslash(-\infty, 0]$ and the parameter $\nu \in \mathbb{C}$. Then the following equalities hold true

$$
R^{\lambda} C_{\nu}(z)=z^{-(\nu-\lambda) / 2} J_{\nu-\lambda, \lambda}^{1}(2 \sqrt{z}) .
$$


Other result, which we consider, refers to the Riemann-Liouville derivative of the multi-index Mittag-Leffler function (13) (for details and proof see [16] and $[19])$.

Theorem 20. Let $z \in \mathbb{C} \backslash(-\infty, 0]$ be a complex variable and let $\alpha_{i}, \beta_{i}, \gamma_{i}, \omega$, $\lambda \in \mathbb{C}, \operatorname{Re}\left(\alpha_{i}\right)>0, \operatorname{Re}\left(\beta_{i_{0}}\right)>0, \operatorname{Re}(\lambda)>0, i=1, \ldots, m, 1 \leq i_{0} \leq m, i_{0} \in \mathbb{N}$, then the following identity holds

$$
D^{\lambda}\left[z^{\beta_{i_{0}}-1} E_{\left(\alpha_{i}\right),\left(\beta_{i}\right)}^{\left(\gamma_{i}\right), m}\left(\omega z^{\alpha_{i_{0}}}\right)\right]=z^{\beta_{i_{0}}-\lambda-1} E_{\left(\alpha_{i}\right),\left(\widetilde{\beta}_{i}\right)}^{\left(\gamma_{i}\right), m}\left(\omega z^{\alpha_{i_{0}}}\right),
$$

with $\widetilde{\beta}_{i}=\beta_{i}$, if $i \neq i_{0}$, and $\widetilde{\beta}_{i_{0}}=\beta_{i_{0}}-\lambda$.

Remark 21. In particular, as a result of (62), we obtain the following identities:

$$
\begin{gathered}
D^{\lambda}\left[z^{\beta-1} E_{\alpha, \beta}^{\gamma}\left(\omega z^{\alpha}\right)\right]=z^{\beta-\lambda-1} E_{\alpha, \beta-\lambda}^{\gamma}\left(\omega z^{\alpha}\right), \\
D^{\lambda}\left[z^{\beta_{i_{0}}-1} E_{\left(\alpha_{i}\right),\left(\beta_{i}\right)}^{m}\left(\omega z^{\alpha_{i_{0}}}\right)\right]=z^{\beta_{i_{0}}-\lambda-1} E_{\left(\alpha_{i}\right),\left(\widetilde{\beta}_{i}\right)}^{m}\left(\omega z^{\alpha_{i_{0}}}\right),
\end{gathered}
$$

with $\widetilde{\beta}_{i_{0}}=\beta_{i_{0}}-\lambda$ and $\widetilde{\beta}_{i}=\beta_{i}$, if $i \neq i_{0}$. These results follow, taking into account (11), if $m=1$, and respectively (12), if $\gamma_{1}=\cdots=\gamma_{m}=1$. The mentioned relations are well known, for which see Refs. [6] and [8].

Theorem 22. Let $z \in \mathbb{C} \backslash(-\infty, 0]$ be a complex variable and let $\nu \in \mathbb{C}$ be a parameter. Then the following equalities hold true

$$
\begin{gathered}
D^{\lambda}\left[z^{-\sigma} J_{\nu, \sigma}^{2, q}(z)\right]=2^{-\lambda} z^{-\sigma} J_{\nu-\lambda, \sigma}^{2, q}(z), \\
D^{\lambda}\left[z^{\nu / 2} J_{\nu, \sigma}^{1, q}(2 \sqrt{z})\right]=z^{\nu / 2-\lambda / 2} J_{\nu-\lambda, \sigma}^{1, q}(2 \sqrt{z}),
\end{gathered}
$$

if additionally $\operatorname{Re}(\sigma+\nu+1)>0$. If $\operatorname{Re}(\sigma+1)>0$, then

$$
D^{\lambda}\left[z^{-\nu / 2} J_{\nu, \sigma}^{\mu, q}(2 \sqrt{z})\right]=z^{\sigma-\lambda} E_{(\mu, 1, \ldots, 1),(\sigma+\nu+1, \sigma-\lambda+1, \sigma+1, \ldots, \sigma+1)}^{q+1}(-z),
$$

Proof. Using (27), the function $z^{-\sigma} J_{\nu, \sigma}^{2, q}(z)$ can be expressed as follows

$$
z^{-\sigma} J_{\nu, \sigma}^{2, q}(z)=2^{-\nu-2 \sigma} z^{\nu+\sigma} \widetilde{J}_{\nu, \sigma}^{2, q}(z)
$$


Then, in view of (15), applying the formula (63) with $\omega=-1 / 4, \alpha_{i_{0}}=2$, and the corresponding $\beta_{i_{0}}=\sigma+\nu+1$, we obtain

$$
D^{\lambda}\left[z^{\sigma+\nu} \widetilde{J}_{\nu, \sigma}^{2, q}(z)\right]=z^{\sigma+\nu-\lambda} \widetilde{J}_{\nu-\lambda, \sigma}^{2, q}(z)=\frac{z^{-\sigma}}{2^{-(\nu+2 \sigma)} 2^{\lambda}} J_{\nu-\lambda, \sigma}^{2, q}(z) .
$$

Now, using (67), the equality (64) immediately follows.

Further, proving (65), we express $z^{\nu / 2} J_{\nu, \sigma}^{1, q}(2 \sqrt{z})$ in an analogical way, and we obtain:

$$
z^{\nu / 2} J_{\nu, \sigma}^{1, q}(2 \sqrt{z})=z^{\nu+\sigma} \widetilde{J}_{\nu, \sigma}^{1, q}(2 \sqrt{z}) .
$$

Then, in view of (16) with $\omega=-1, \alpha_{i_{0}}=1$, and $\beta_{i_{0}}=\sigma+\nu+1$, we can write down that

$$
D^{\lambda}\left[z^{\sigma+\nu} \widetilde{J}_{\nu, \sigma}^{1, q}(2 \sqrt{z})\right]=z^{\sigma+\nu-\lambda} \widetilde{J}_{\nu-\lambda, \sigma}^{1, q}(2 \sqrt{z})=z^{\nu / 2-\lambda / 2} J_{\nu-\lambda, \sigma}^{1, q}(2 \sqrt{z})
$$

from where

$$
D^{\lambda}\left[z^{\nu / 2} J_{\nu, \sigma}^{1, q}(2 \sqrt{z})\right]=z^{\nu / 2-\lambda / 2} J_{\nu-\lambda, \sigma}^{1, q}(2 \sqrt{z})
$$

In order to prove (66), we apply the formula (63) for $\omega=-1, \alpha_{i_{0}}=1$, and the corresponding $\beta_{i_{0}}=\sigma+1$. Then, following the same idea as above, we obtain consequently

$$
\begin{gathered}
z^{-\nu / 2} J_{\nu, \sigma}^{\mu, q}(2 \sqrt{z})=z^{-\nu / 2} z^{\nu / 2+\sigma} \widetilde{J}_{\nu, \sigma}^{\mu, q}(2 \sqrt{z})=z^{\sigma} \widetilde{J}_{\nu, \sigma}^{\mu, q}(2 \sqrt{z}), \\
=z^{\sigma} \sum_{k=0}^{\infty} \frac{(-z)^{k}}{\Gamma(\mu k+\sigma+\nu+1)(\Gamma(k+\sigma+1))^{q}} .
\end{gathered}
$$

Therefore

$$
\begin{gathered}
D^{\lambda}\left[z^{-\nu / 2} J_{\nu, \sigma}^{\mu, q}(2 \sqrt{z})\right]=D^{\lambda}\left[z^{\sigma} \widetilde{J}_{\nu, \sigma}^{\mu, q}(2 \sqrt{z})\right] \\
=z^{\sigma-\lambda} \sum_{k=0}^{\infty} \frac{(-z)^{k}}{\Gamma(\mu k+\sigma+\nu+1) \Gamma(k+\sigma-\lambda+1)(\Gamma(k+\sigma+1))^{q-1}} \\
=z^{\sigma-\lambda} E_{(\mu, 1, \ldots, 1),(\sigma+\nu+1, \sigma-\lambda+1, \sigma+1, \ldots, \sigma+1)}^{q+1}(-z),
\end{gathered}
$$

which proves (66).

Remark 23. Putting $q=1$ in the equalities (64), (65) and (66), they give the corresponding results for the corresponding 3-parametric functions (5) and (6). 
In particular, if $q=1$ and $\sigma=0$, the formula (65) produces a result for the classical Bessel function. Namely, the following corollary can be formulated.

Corollary 24. Let $z \in \mathbb{C} \backslash(-\infty, 0]$ be a complex variable and let $\nu$ be the parameter, satisfying the conditions $\nu \in \mathbb{C}, \operatorname{Re}(\nu+1)>0$. Then the following equalities hold true

$$
D^{\lambda}\left[z^{\nu / 2} J_{\nu}(2 \sqrt{z})\right]=z^{\nu / 2-\lambda / 2} J_{\nu-\lambda, 0}^{1,1}(2 \sqrt{z})=z^{\nu / 2-\lambda / 2} J_{\nu-\lambda}(2 \sqrt{z}) .
$$

Theorem 25. Let $z \in \mathbb{C} \backslash(-\infty, 0]$ be a complex variable and let $\mu, \nu$ be the parameters, satisfying the conditions $\nu \in \mathbb{C}, \mu>0$. Then the following equalities hold true

$$
D^{\lambda}\left[J_{\nu}^{\mu}(z)\right]=z^{-(\nu+\lambda) / 2} J_{\nu+\lambda,-\lambda}^{\mu}(2 \sqrt{z}),
$$

and

$$
D^{\lambda}\left[z^{\nu} C_{\nu}(z)\right]=z^{\nu-\lambda} C_{\nu-\lambda}(z), \quad \text { for } \quad \operatorname{Re}(\nu+1)>0 .
$$

Proof. Expressing $J_{\nu}^{\mu}(z)$ from (12) with $\alpha_{1}=1, \beta_{1}=1, \alpha_{2}=\mu$ and $\beta_{2}=\nu+1$, and applying after that the second formula (63) with $\alpha_{i_{0}}=1$, $\beta_{i_{0}}=1$ and $\omega=-1$, we obtain

$$
\begin{aligned}
D^{\lambda}\left[J_{\nu}^{\mu}(z)\right]= & z^{-\lambda} \sum_{k=0}^{\infty} \frac{(-z)^{k}}{\Gamma(k-\lambda+1) \Gamma(\mu k+\nu+1)} \\
& =z^{-\lambda} \widetilde{J}_{\nu+\lambda,-\lambda}^{\mu}(2 \sqrt{z}) .
\end{aligned}
$$

Then, in view of $(27)$, it follows

$$
D^{\lambda}\left[J_{\nu}^{\mu}(z)\right]=z^{-(\nu+\lambda) / 2} J_{\nu+\lambda,-\lambda}^{\mu}(2 \sqrt{z}),
$$

which proves (73).

Further setting $\mu=1$ in $J_{\nu}^{\mu}$ gives $C_{\nu}$. Applying again the second formula (63) with $\alpha_{i_{0}}=1$ and $\beta_{i_{0}}=\nu+1$ leads to the following result

$$
\begin{aligned}
D^{\lambda}\left[z^{\nu} C_{\nu}(z)\right]= & z^{\nu-\lambda} \sum_{k=0}^{\infty} \frac{(-z)^{k}}{\Gamma(k+1) \Gamma(k+\nu-\lambda+1)} \\
& =z^{\nu-\lambda} C_{\nu-\lambda}(z) .
\end{aligned}
$$

Thus the correctness of (74) follows. 
In particular, taking $\mu=1$, Theorem 25 gives a result referring to the classical Bessel-Clifford functions, i.e. we produce the following corollary.

Corollary 26. Let $z \in \mathbb{C} \backslash(-\infty, 0]$ be a complex variable and the parameter $\nu \in \mathbb{C}$. Then the following equality holds true

$$
D^{\lambda} C_{\nu}(z)=z^{-(\nu+\lambda) / 2} J_{\nu+\lambda,-\lambda}^{1}(2 \sqrt{z}) .
$$

Another interesting result, also connected with the differentiation of an arbitrary order, is given by the theorem below.

Theorem 27. Let $\alpha_{i}, \beta_{i}, \widetilde{\lambda} \in \mathbb{C}$ and $\operatorname{Re}_{\widetilde{\sim}}\left(\alpha_{i}\right)>0$ for $i=1, \ldots, m$, and let also $0<\operatorname{Re}(\widetilde{\lambda})<1, n \in \mathbb{N}$, and $\lambda=n-1+\widetilde{\lambda}$. Then it holds

$$
D^{\lambda}\left[z^{\tilde{\lambda}} E_{\left(\alpha_{i}\right),\left(\beta_{i}\right)}(w z)\right]=w^{n-1} \Gamma(\lambda+1) E_{\left(\alpha_{i}\right),\left(\widetilde{\beta}_{i}\right)}^{\left(\gamma_{i}\right)}(w z) \quad(\text { for }|\arg z|<\pi),
$$

with parameters $\widetilde{\beta}_{i}=\beta_{i}+(n-1) \alpha_{i}$ and $\gamma_{1}=\lambda+1, \gamma_{2}=\cdots=\gamma_{m}=1$.

Proof. The proof of this theorem is omitted, because it follows completely the same idea as that for $w=1$ (for details see [20]).

Further, if $0<\operatorname{Re}(\lambda)<1$, then $n=1, \omega^{n-1}=1, \widetilde{\lambda}=\lambda$ and $\widetilde{\beta}_{i}=\beta_{i}$. Then the following corollary can be produced.

Corollary 28. Let $z \in \mathbb{C} \backslash(-\infty, 0]$ be a complex variable and the parameters $\nu \in \mathbb{C}, \alpha_{i}, \beta_{i}, \tilde{\lambda} \in \mathbb{C}$ and $\operatorname{Re}\left(\alpha_{i}\right)>0$ for $i=1, \ldots, m$, and let also $0<\operatorname{Re}(\lambda)<1$. Then the following equality hold true

$$
D^{\lambda}\left[z^{\lambda} E_{\left(\alpha_{i}\right),\left(\beta_{i}\right)}(w z)\right]=\Gamma(\lambda+1) E_{\left(\alpha_{i}\right),\left(\beta_{i}\right)}^{\left(\gamma_{i}\right)}(w z) \quad(\text { for }|\arg z|<\pi),
$$

with parameters $\gamma_{1}=\lambda+1, \gamma_{2}=\cdots=\gamma_{m}=1$.

Remark 29. If $m=1$ in the equalities (78) and (79), then they give the corresponding relations for the functions (11) and the derivative of order $\lambda$ of the function (3). More precisely, (78) and (79) are reduced to

$$
D^{\lambda}\left[z^{\tilde{\lambda}} E_{\alpha, \beta}(w z)\right]=w^{n-1} \Gamma(\lambda+1) E_{\alpha, \widetilde{\beta}}^{\gamma}(w z) \quad(\text { for }|\arg z|<\pi),
$$


with parameters $\widetilde{\beta}=\beta+(n-1) \alpha$ and $\gamma=\lambda+1$, respectively

$$
D^{\lambda}\left[z^{\lambda} E_{\alpha, \beta}(w z)\right]=\Gamma(\lambda+1) E_{\alpha, \beta}^{\gamma}(w z) \quad(\text { for }|\arg z|<\pi),
$$

with parameter $\gamma=\lambda+1$.

Theorem 30. Let $\alpha_{i}, \beta_{i}, \lambda \in \mathbb{C}$ and $\operatorname{Re}\left(\alpha_{i}\right)>0$ for $i=1, \ldots, m$. Moreover, let $0<\operatorname{Re}(\lambda)<1$. Then

$$
R^{\lambda}\left[z^{-\lambda} E_{\left(\alpha_{i}\right),\left(\beta_{i}\right)}(\omega z)\right]=\Gamma(1-\lambda) E_{\left(\alpha_{i}\right),\left(\beta_{i}\right)}^{\left(\gamma_{i}\right)}(\omega z) \quad(\text { for }|\arg z|<\pi),
$$

with parameters $\gamma_{1}=1-\lambda, \gamma_{2}=\cdots=\gamma_{m}=1$.

The theorem is proved in [20]. As a corollary, taking $m=1$ in Theorem 30, the corresponding result for the functions (3) and (11) can be formulated.

Corollary 31. Let $\alpha, \beta, \lambda \in \mathbb{C}$, and let $\operatorname{Re}(\alpha)>0$ and $0<\operatorname{Re}(\lambda)<1$. Then

$$
R^{\lambda}\left[z^{-\lambda} E_{\alpha, \beta}(\omega z)\right]=\Gamma(1-\lambda) E_{\alpha, \beta}^{1-\lambda}(\omega z) \quad(\text { for }|\arg z|<\pi) .
$$

Now, the corresponding results, referring to the Bessel type functions can be obtained due to the relations in Section 4, and they are given below.

Theorem 32. Let $\alpha_{i}, \beta_{i}, \lambda \in \mathbb{C}$ and $\operatorname{Re}\left(\alpha_{i}\right)>0$ for $i=1, \ldots, m$. Moreover, let $0<\operatorname{Re}(\lambda)<1$. Then

$$
\begin{gathered}
R^{\lambda}\left[z^{-\lambda} J_{\nu}^{\mu}(z)\right]=\Gamma(1-\lambda) E_{(\mu, 1)(\nu+1,1)}^{(1-\lambda, 1)}(-z) \\
=\Gamma(1-\lambda) \sum_{k=0}^{\infty} \frac{(1-\lambda)_{k}(1)_{k}}{k ! \Gamma(\mu k+\nu+1)} \frac{(-z)^{k}}{(k !)^{2}} \quad(\text { for }|\arg z|<\pi) .
\end{gathered}
$$

Proof. Using the formulae (19) and (82), we have

$$
\begin{aligned}
& R^{\lambda}\left[z^{-\lambda} J_{\nu}^{\mu}(z)\right]=R^{\lambda}\left[z^{-\lambda} E_{(\mu, 1)(\nu+1,1)}(-z)\right] \\
&=\Gamma(1-\lambda) E_{(\mu, 1)(\nu+1,1)}^{(1-\lambda, 1)}(-z) \\
&=\Gamma(1-\lambda) \sum_{k=0}^{\infty} \frac{(1-\lambda)_{k}(1)_{k}}{k ! \Gamma(\mu k+\nu+1)} \frac{(-z)^{k}}{(k !)^{2}}
\end{aligned}
$$

which proves the theorem. 
In the particular case, if $\mu=1$ and $\nu=-\lambda$, Theorem 32 produces the following corollary for the Bessel-Clifford functions.

Corollary 33. Let $\alpha_{i}, \beta_{i}, \lambda \in \mathbb{C}$ and $\operatorname{Re}\left(\alpha_{i}\right)>0$ for $i=1, \ldots, m$. Moreover, let $0<\operatorname{Re}(\lambda)<1$. Then

$$
R^{\lambda}\left[z^{-\lambda} C_{-\lambda}(z)\right]=C_{0}(z) \quad(\text { for }|\arg z|<\pi) .
$$

Proof. Using the formulae (19), (20) and (84), we have

$$
\begin{gathered}
R^{\lambda}\left[z^{-\lambda} C_{-\lambda}(z)\right]=R^{\lambda}\left[z^{-\lambda} J_{-\lambda}^{1}(z)\right] \\
=\Gamma(1-\lambda) \sum_{k=0}^{\infty} \frac{(1-\lambda)_{k}(1)_{k}}{k ! \Gamma(k+1-\lambda)} \frac{(-z)^{k}}{(k !)^{2}}=\sum_{k=0}^{\infty} \frac{(-z)^{k}}{(k !)^{2}}=C_{0}(z),
\end{gathered}
$$

which proves the corollary.

In conclusion, we can summarize that this survey paper refers to various differential and integral relations in the class of the multi-index Mittag-Leffler functions. More precisely, a part of them connects the derivatives and integrals of the 2m-parametric Mittag-Leffler functions with the $3 m$-parametric MittagLeffler functions, and the other part is between functions with the same number of indices. As special cases the relations are for different representatives of this class, all of them of Bessel type. In general the result of differentiation and integration of a multi-index Mittag-Leffler function is also a function of the same class (up to a constant and a power function). However, for the Bessel type functions the facts are sometimes different. There are three groups of results given.

The first group consists of relations, where the results of differentiations and integrations are expressed by multi-index Mittag-Leffler functions but they in particular are not of Bessel type. In the second group the relations are between Bessel type functions with different number of indices, and in the third group they are with the same number of indices.

There are obtained interesting relations between the integrals and derivatives of the 4-index Bessel type functions $J_{\nu, \sigma}^{\mu, q}$, given by (7) and (8), on the one hand, and the multi-index Mittag-Leffler functions (12) and (13) on the other hand, and also between (7) and (8) and the corresponding high-order derivatives and fractional Riemann-Liouville integrals and derivatives of them. In particular, for $q=1$ the results refer to the 3 -index functions (5) and (6). We emphasize 
that there are given simple relations between the 3-index generalized BesselMaitland functions (6) and the corresponding fractional Riemann-Liouville integrals and derivatives of the 2-index Bessel-Maitland functions $J_{\nu}^{\mu}(z)$, defined by (4). More precisely these integrals and derivatives are expressed by the 3index functions (6), up to matching power functions. Besides, the derivatives of integer order $n$ of (4) can also be expressed by a function of the kind (4), multiplied by the constant $(-1)^{n}$. Finally, the results for the classical Bessel and Bessel-Clifford functions merely follow as corollaries.

\section{Acknowledgments}

This paper is performed in the frames of the Bilateral Res. Projects 'Analytical and numerical methods for differential and integral equations and mathematical models of arbitrary (fractional or high integer) order' between BAS and SANU and 'Analysis, Geometry and Topology' between BAS and MANU.

\section{References}

[1] E. Bazhlekova, I. Dimovski, Exact solution for the fractional cable equation with nonlocal boundary conditions, Central Europ. J. of Phys., 11, No 10 (2013), 1304-1313; DOI: 10.2478/s11534-013-0213-5.

[2] E. Bazhlekova, Subordination in a class of generalized time-fractional diffusion-wave equations, Fract. Calc. Appl. Anal., 21, No 4 (2018), 869900, DOI: 10.1515/fca-2018-0048.

[3] A.A. Kilbas, A.A. Koroleva, Generalized Mittag-Leffler function and its extension, Tr. Inst. Mat. Minsk, 13, No 1 (2005), 43-52 (In Russian).

[4] A.A. Kilbas, A.A. Koroleva, Integral transform with the extended generalized Mittag-Leffler function, Math. Modeling and Anal., 11, No 2 (2006), 173-186.

[5] A.A. Kilbas, A.A. Koroleva, S.V. Rogosin, Multi-parametric Mittag-Leffler functions and their extension, Fract. Calc. Appl. Anal., 16, No 2 (2013), 378-404; DOI: 10.2478/s13540-013-0024-9.

[6] A.A. Kilbas, M. Saigo, R.K. Saxena, Generalized Mittag-Leffler function and generalized fractional calculus operators, Integr. Transf. Spec. Funct., 15, No 1 (2004), 31-49. 
[7] V. Kiryakova, Generalized Fractional Calculus and Applications, Longman Sci. Tech. \& J. Wiley, Harlow - N. York (1994).

[8] V. Kiryakova, Multiple (multiindex) Mittag-Leffler functions and relations to generalized fractional calculus, J. of Comput. and Appl. Math., 118 (2000), 241-259, doi: 10.1016/S0377-0427(00)00292-2.

[9] V. Kiryakova, The special functions of fractional calculus as generalized fractional calculus operators of some basic functions, Computers and Math. with Appl., 59, No 3 (2010), 1128-1141; doi: 10.1016/j.camwa.2009.05.014.

[10] V. Kiryakova, From the hyper-Bessel operators of Dimovski to the generalized FC, Fract. Calc. Appl. Anal., 17, No 4 (2014), 977-1000; DOI: 10.2478/s13540-014-0210-4.

[11] V. Kiryakova, Fractional calculus operators of special functions? - The result is well predictable!, Chaos, Solitons \& Fractals, 102 (2017), 2-15; doi: $10.1016 /$ j.chaos.2017.03.006.

[12] V. Kiryakova, Yu. Luchko, The multi-index Mittag-Leffler functions and their applications for solving fractional order problems in applied analysis, In: American Institute of Physics - Conf. Proc., 1301 (2010), 597-613; doi: $10.1063 / 1.3526661$.

[13] Yu. Luchko, Asymptotics of zeros of the Wright function, J. for Analysis and its Applications, 19 (2000), No 1, 1-12.

[14] Yu. Luchko, Algorithms for evaluation of the Wright function for the real arguments' values, Fract. Calc. Appl. Anal., 11, No 1 (2008), 57-75.

[15] J.A.T. Machado, V. Kiryakova, The chronicles of Fractional Calculus, Fract. Calc. Appl. Anal., 20, No 2 (2017), 307-336; DOI: 10.1515/fca2017-0017.

[16] J. Paneva-Konovska, Multi-index (3m-parametric) Mittag-Leffler functions and fractional calculus, Compt. rend. Acad. bulg. Sci., 64, No 8 (2011), 1089-1098.

[17] J. Paneva-Konovska, Periphery behaviour of series in Mittag-Leffler type functions, I, Intern. J. Appl. Math., 29, No 1 (2016), 69-78; doi: 10.12732/ijam.v29i1.6. 
[18] J. Paneva-Konovska, Periphery behaviour of series in Mittag-Leffler type functions, II, Intern. J. Appl. Math., 29, No 2 (2016), 175-186; doi: 10.12732/ijam.v29i2.2.

[19] J. Paneva-Konovska, From Bessel to Multi-Index Mittag Leffler Functions: Enumerable Families, Series in them and Convergence, World Scientific Publ., London (2016); doi: 10.1142/q0026.

[20] J. Paneva-Konovska, Differential and integral relations in the class of multiindex Mittag-Leffler functions, Fract. Calc. Appl. Anal., 21, No 1 (2018), 254-265; DOI: 10.1515/fca-2018-0016.

[21] J. Paneva-Konovska, Bessel type functions: Relations with integrals and derivatives of arbitrary orders, In: American Institute of Physics - Conf. Proc., 2048 (2018), Art. 050015, 050015-1-050015-6; doi: $10.1063 / 1.5082114$.

[22] R.S. Pathak, Certain convergence theorems and asymptotic properties of a generalization of Lommel and Maitland transformations, Proc. Nat. Acad. Sci. India, A-36, No 19 (1966), 81-86.

[23] T.R. Prabhakar, A singular equation with a generalized Mittag-Leffler function in the kernel, Yokohama Math. J., 19 (1971), 7-15.

[24] A.I. Prieto, S.S. de Romero, H.M. Srivastava, Some fractional-calculus results involving the generalized Lommel-Wright and related functions, Appl. Math. Letters, 20 (2007), 17-22.

[25] S. Samko, A. Kilbas, O. Marichev, Fractional Integrals and Derivatives. Theory and Applications, Gordon and Breach, N. York - London (1993).

[26] E.M. Wright, On the coeficients of power series having exponential singularities, J. London Math. Soc., 8 (1933), 71-79.

[27] E.M. Wright (1940), The generalized Bessel function of order greater than one, Quart. J. Math., Oxford Ser., 11, 36-48.

[28] S. Yakubovich, Yu. Luchko, The Hypergeometric Approach to Integral Transforms and Convolutions, Kluwer Acad. Publ., Dordrecht - Boston - London (1994). 